\section{Defective viruses to blame?}

\section{Robin A. Weiss}

THREE new papers on an acquired immune deficiency syndrome in mice caused by a strain of murine leukaemia virus suggest that a defective genome is responsible for the immunodeficiency. Janet Hartley, Sandy Morse and colleagues ${ }^{1}$ at the National Institutes of Health have found an unusual pattern of genetic susceptibility to murine AIDS consistent with a defective genome being carried by different replication-competent 'helper' viruses. This has been followed up by the molecular identification, also by Hartley and colleagues $^{2}$, of the defective virus. And an apparently identical genome has been independently identified by Paul Jolicoeur and colleagues in Montreal, as they report on page 505 of this issue ${ }^{3}$. After cloning of the defective virus, which retains gag sequences in its 4.8-kilobase genome, and rescue by non-pathogenic helper viruses, inoculation into mice leads to the induction of AIDS $^{2,3}$. These findings supplement that from Jim Mullins's group ${ }^{4}$ which shows that in cats, immunodeficiency can be caused by a defective feline leukaemia virus.

Many classes of animal RNA virus accumulate defective variants when propagated serially at high multiplicities of infection. This was first observed by von Magnus with influenza virus ${ }^{5}$. Such defective viruses frequently interfere with the replication of full-length, non-defective genomes and they may play a role in pathogenesis and persistent infection ${ }^{6}$. Defective retroviruses, however, do not typically interfere with their helper viruses and are not simply truncated forms of the competent virus; they contain recombinant viral or cellular sequences that can directly influence the pathogenesis of the virus population.

\section{Retroviral oncogenes}

The notion that defective variants of retroviruses cause specific disease was raised more than 25 years ago, when Hanafusa et al. $^{7}$ showed that the Bryan strain of Rous sarcoma virus was defective. This classical study led eventually to the identification of oncogenes derived from cellular genes, usually by insertion at the expense of viral genes, thereby rendering the viral genome replicationdefective. Nearly all retroviral oncogenes are carried in defective viruses requiring helper viruses for transmission.

That defective retroviruses can carry 'scrambled' viral genes determining new disease patterns, rather than cellular genes, was first seen with the spleen-focus-forming virus (SFFV) component of Friend erythroid leukaemia virus. The defective SFFV genome encodes an incomplete retroviral env gene derived from exogenous, 'ecotropic' and endogenous, 'xenotropic' sequences. This new form of glycoprotein probably interacts with specific 'dual-tropic' receptors on erythroid precursor cells to trigger their proliferation ${ }^{8}$. As the term 'viral oncogene' is usually restricted to gene sequences of mainly cellular origin, and of course pertains to those genes inducing neoplastic transformation, we need a new term for retroviral genes of viral origin specifying particular disease forms - I would offer as a suggestion 'pathogenes' with path as the generic equivalent to onc.

The SFFV gene determining erythroleukaemia is derived from env sequences, as is the gene inducing feline AIDS in defective feline leukaemia virus ${ }^{4}$. A recent paper by Poss et al. ${ }^{9}$ indicates that the property of the env protein of the defective feline AIDS virus which makes it cytopathic for $T$ cells lies in its post-translational glycosylation pattern. With murine AIDS, however, it appears that the functional path gene in the defective virus is derived from the gag gene. Aziz, Hanna and Jolicoeur ${ }^{3}$ have sequenced the 4.8kilobase genome and found four open reading frames, two corresponding to portions of $g a g$, one to part of $p o l$ and one to part of pol-env.

The p12 region of the largest open reading frame (586 amino acids) has only 40-50-per-cent identity with Gag p12 of other murine retroviruses, and it is this product that the authors suspect is responsible for inducing AIDS. Chattopadhyay et $a l^{2}$ also implicate a gag-related product because Gag antisera including anti-p12, but not anti-Pol and anti-Env sera, immunoprecipitate a 60 -kilodalton protein from a murine cell line carrying the defective genome in the absence of helper virus.

The study of defective murine and feline leukaemia viruses causing immune deficiency syndromes should enhance our understanding of the various ways retroviruses induce disease. Being oncoviruses rather than lentiviruses and therefore less closely related to the human immunodeficiency virus (HIV), their use in screening anti-viral drugs is doubtful; if one wishes to use an oncovirus for this purpose, Friend virus induces disease in mice more quickly with a more quantifiable endpoint.

Aziz et al. ${ }^{3}$ cite striking similarities of murine AIDS to human AIDS, though there are striking differences too. The disease was first described as lymphoma ${ }^{10}$, owing to what is now believed to be a polyclonal B-cell hyperplasia rapidly induced by the virus. A detailed study ${ }^{11}$ of the syndrome showed that a profound suppression of humoral and cellular immunity soon follows the lymphoproliferative phase. Both the B-cell hyperplasia and the immune suppression seem to be T-cell dependent, because the virus affects nude mice much less severely'2. However, it is not clear that the virus infects T-helper cells, and the macrophage reservoir and neurotropism characteristic of HIV have not been examined in murine AIDS. In my view. the diseases induced by HIV-related, T-lymphotropic lentiviruses of cats (FIV), cattle (BIV) and monkeys (SIV) will prove to be more proximate models of human AIDS.

\section{Role in human AIDS}

Nonetheless, the discovery of defective oncoviruses inducing severe immunosuppression in cats and mice raises the question whether defective HIV genomes might play a role in human AIDS. Replication-competent, molecularly cloned HIV and SIV genomes have not yet been shown to induce AIDS, and even if they do, that might require the generation of defective, variant genomes during the long incubation period between infection and manifestations of disease.

Several HIV laboratories are finding a myriad of viral forms when sequences are amplified directly from infected tissues, rather than selecting for replicationcompetent viruses in cell culture. Simon Wain-Hobson (personal communication) refers to such HIV populations as "quasispecies' because they represent greater diversity than might be expected from classical population variability. The mixture of defective oncoviruses and the helper viruses required for their generation and transmission represents a special case of quasi-species genome dynamics. No doubt defective forms of HIV will be carefully scrutinized at the molecular level.

Robin A. Weiss is at the Chester Beatty Laboratories, Institute of Cancer Research, London SW3 6JB, UK.

1. Hartley, J.W., Frederickson. T.N., Yetter, R.A., Makino, M. \& Morse, H.C. J. Virol, 63, 1223-1231 (1989)

2. Chattopadhyay, S.K., Morse, H.C., Makino, M. Ruscetti, S.K. \& Hartley, J.W. Proc. natn. Acad. Sci. U.S.A. (in the press).

3. Aziz, D.C., Hanna, Z. \& Jolicoeur, P. Nature 338, 505 $508(1989)$.

4. Overbaugh, J., Donahue, P.R., Quackenbush, S.L. Hoover, E.A. \& Mullins, J.I. Science 239. 906-910 (1988)

5. Von Magnus, P. Acta path. microbiol. immun. scand. 28 278-293 (1951).

6. Huang, A.S. \& Baltimore, D. Nature 226, 325-327 (1970)

7. Hanafusa, H., Hanafusa, T. \& Rubin, H. Proc natn Acad. Sci. U.S.A. 49, 572-580 (1963)

8. Li, J.P.. Bestwick, R. K., Spiro, C. \& Kabat. D. J. Virol, 61 2782-2792 (1987).

. Poss, M.L.. Mullins, J.I. \& Hoover, E.A. J. Virol. 63, 189 195 (1989).

10. Haas. M. \& Reshef, T. Eur. J. Cancer 16, 909-913 (1980)

11. Mosier, D.E., Yetter, R.A. \& Morse, H.C. J. exp. Med 161, 766-784 (1985)

12. Mosier, D.E., Yetter. R.A. \& Morse. H.C. J. exp. Med. 165, 1737-1741 (1987) 